\title{
Comparing the values of progesterone in the blood of bitches as measured with a chemiluminescence immunoassay and a radioimmunoassay
}

\author{
J.O. Nöthling ${ }^{a} *$ \\ K.G.M. De Cramer ${ }^{\text {a,1 }}$ \\ a Section of Reproduction, Department of Production Animal Studies, Faculty of Veterinary Science, Private \\ Bag X04, Onderstepoort 0110, South Africa \\ * Corresponding author. Tel +27 0125298020. Section of Reproduction, Department of Production Animal \\ Studies, Faculty of Veterinary Science, University of Pretoria, Private Bag X04, Onderstepoort, 0110, South \\ Africa. Email address: johan.nothling@up.ac.za. \\ ${ }^{1}$ Present address: Rant en Dal Animal Hospital, 51 Cecil Knight Street, Mogale City, Gauteng, South Africa, \\ kdcramer@mweb.co.za
}

\section{Abstract}

A ${ }^{125}$ I radioimmunoassay (RIA) has long been used to determine the value of progesterone in serum or plasma of bitches but was discontinued in 2014. A chemiluminescence immunoassay (CLIA) gained prominence since 2003 to determine the value of progesterone in serum of bitches but the assay changed in 2012. This study assessed the agreement between progesterone values obtained with RIA in plasma (progRIA) and with the post-2012 CLIA (progCLIA) in the serum of bitches. ProgCLIA was determined in 110 serum samples from 40 bitches in pro-oestrus or early oestrus and compared to progRIA in plasma samples collected from the same bitches at the same time, where progRIA had a uniform distribution between 0.5 and $25 \mathrm{nmol} / \mathrm{L}$. Two replicate analyses of each serum or plasma sample were simultaneously done in the same assay. For RIA and CLIA, the intra-assay CVs were $5.85 \%$ and $6.70 \%$ and the interassay CVs $8.45 \%$ and 9.16\%. For RIA and CLIA the progesterone values obtained with replicate analyses differed by as much as $11 \%-31 \%$ in $25 \%$ of samples. On average, the value of progCLIA was $85 \%$ of that of progRIA ( $95 \%$ CI $58 \%-112 \%, n=110)$, with $88 \%$ of progCLIAs being lower than the progRIAs. This study shows that RIA and CLIA may yield replicate 
values that differ by as much as $11 \%-30 \%$ in about a quarter of samples analysed, necessitating replicate analyses if precise values are required. The study provides an equation by which to estimate progCLIA from progRIA.

Keywords: progesterone, bitch, radioimmunoassay, Coat-A-Count, Immulite,

\section{Introduction}

In bitches, precise and accurate values of progesterone in blood plasma (plasma) or serum is the key element for various clinical decisions. One such decision is whether a bitch is likely to be within $36 \mathrm{hr}$ (Concannon, Butler, Hansel, Knight, \& Hamilton, 1978) or $30 \mathrm{hr}$ (Baan et al., 2005) from whelping. Another decision is to determine whether a bitch is in anoestrus or still in late dioestrus (Chu, Lee, \& Wright, 2006; Concannon, 2011; Jeffcoate, 1993). Other decisions relate to determining the optimal breeding time, for example whether a bitch is likely to be at the onset of the LH surge (Concannon, 2011) or at the LH peak (Bergeron, Nykamp, Brisson, Madan, \& Gartley, 2013; Concannon, Hansel, \& McEntee, 1977; Concannon, Hansel, \& Visek, 1975) or at ovulation (Fontbonne, 2008; Groppetti et al., 2015; Schmicke, Urhausen, Wolf, Schmidt, \& Günzel-Apel, 2016). Starting before ovulation, but more so thereafter, the value of progesterone not only increases rapidly but its value at comparable times across bitches vary progressively more as the time since ovulation increases. So, for example, the value of progesterone in plasma varied from 5.4 to $11.8 \mathrm{nmol} / \mathrm{L}$ at the time of the $\mathrm{LH}$ peak (Concannon et al., 1977), from 9.5 to $31.2 \mathrm{nmol} / \mathrm{L}$ at ovulation (Fontbonne, 2008), from 29 to $83 \mathrm{nmol} / \mathrm{L}$ at the time of fertilization and from 48 to $102 \mathrm{nmol} / \mathrm{L}$ at the onset of cytological dioestrus (Badinand, Fontbonne, Maurel, \& Siliart, 1993) and from 47 to $254 \mathrm{nmol} / \mathrm{L}$ at the peak during early dioestrus (Concannon, 2011). This increase in variation in progesterone values is due to variation among bitches in the rate of increase in progesterone values over time. From this 
follows that, in order to better estimate the time for breeding, it is more important for an assay to measure the value of progesterone precisely and accurately at values associated with ovulation or lower. An assay with high precision at values associated with ovulation or below will also be beneficial to estimate proximity to parturition and the confirmation of anoestrus.

A ${ }^{125}$ I radioimmunoassay (Coat-A-Count), abbreviated as RIA, has been validated for the determination of the value of progesterone in the serum (Reimers et al., 1991) or plasma (Okkens et al., 2001) of bitches. The assay has been used for decades to determine the value of progesterone in plasma of bitches (Bouchard, Solorzano, Concannon, Youngquist, \& Bierschwal, 1991; de Gier, Kooistra, Djajadiningrat-Laanen, Dieleman, \& Okkens, 2006; Gerstenberg \& Nöthling, 1995; Luz, Bertan, Binelli, \& Lopes, 2006; Okkens et al., 2001; Reimers et al., 1991; Srikandakumar et al., 1986; Steckler, Nöthling, \& Harper, 2013; van Klaveren, Kooistra, Dieleman, Lith, \& Schaefers-Okkens, 2001). The assay has also been used to determine the value of progesterone in serum of bitches (Kutzler, Mohammed, Lamb, \& Meyers-Wallen, 2003; Volkmann, 2006).

During the past 14 years, papers appeared that report studies in which a chemiluminescent immunoassay (Immulite), abbreviated as CLIA, has been used for measuring progesterone in the serum of bitches (Chapwanya, Clegg, Stanley, \& Vaughan, 2008; Kutzler et al., 2003; Rota, Charles, Starvaggi Cucuzza, \& Pregel, 2015; Schmicke et al., 2016; Volkmann, 2006).

Progesterone values obtained with CLIA has been compared to those obtained with RIA (Kutzler et al., 2003; Volkmann, 2006). Chapwanya et al. (2008) compared CLIA to a radioimmunoasssay described by Hoffmann, Kyrein, and Ender (1973). In these studies serum samples were used that contained progesterone at values of $1.56-71 \mathrm{nmol} / \mathrm{L}$ 
(Kutzler et al., 2003), near 0-95 nmol/L in Volkmann (2006) and 0.7-102 nmol/L (Chapwanya et al., 2008). These values extended well above those expected at ovulation or earlier during the oestrous cycle, or those expected near parturition. These studies reported strong, positive, linear correlations between the values determined by CLIA and RIA. What appears as strong linearity over such a large range in progesterone values may mask weak linearity among values measured with radioimmunoassay and CLIA lying near the low, clinically important end of the range included in the correlation analysis (Fisher \& van Belle, 1993).

A linear regression showed that the value of progesterone measured by CLIA in the serum of bitches was expected to be equal to $95.1 \%$ of the value measured with RIA in nmol/L, minus $0.51 \mathrm{nmol} / \mathrm{L}$ (Kutzler et al., 2003). This regression suggests that the value of progesterone measured with CLIA is expected to be lower than that measured by RIA, and that the difference will be larger at low values of progesterone: for a value of $31.8 \mathrm{nmol} / \mathrm{L}$ (RIA), CLIA would yield a value equal to $93.4 \%$ thereof whereas, for a physiologically low value of $3.18 \mathrm{nmol} / \mathrm{L}$ (RIA), CLIA is expected to yield a value measured with CLIA equal to $79 \%$ thereof. Volkmann (2006) reported that the value of progesterone in the serum of bitches, measured with CLIA was about two-thirds of that measured in serum with RIA over the range of values assessed, which is lower than what had been reported in Kutzler et al. (2003). In contrast with these two studies, Chapwanya et al. (2008) reported that CLIA showed higher values than the radioimmunoassay that they used: on average, the values with CLIA were $2.19 \mathrm{nmol} / \mathrm{ml}$ higher than those with RIA. Chapwanya et al. did however find a strong linear correlation $(r=0.99)$ between the two.

Since the studies by Kutzler et al. (2003), Volkmann (2006) and Chapwanya et al. (2008) the two-cycle competitive immunoassay-employing a bead coated with monoclonal mouse anti-progesterone antibodies - that they used (Immulite, Catalogue number 
LKPG1), was changed to a one-cycle competitive immunoassay employing a bead coated with polyclonal rabbit anti-progesterone antibodies, referred to by catalogue number LKPW1 (Ludewig et al., 2012; Schmicke et al., 2016). Schmicke et al. (2016) reported that the new, one-cycle CLIA showed distinctly lower values of progesterone than the older two-cycle CLIA. They used the one-cycle CLIA at the time of ovulation in seven bitches and showed serum progesterone values from 6.4 to $14.3 \mathrm{nmol} / \mathrm{L}$ (mean $10.8 \mathrm{nmol} / \mathrm{L}, S D 2.86$ ), which is distinctly lower than the reference values of $16-$ $25.4 \mathrm{nmol} / \mathrm{L}$ that they used. Their reference values correspond to those reported at the time of ovulation (Fontbonne, 2008) or in various studies $48 \mathrm{hr}$ after the LH peak (Bouchard et al., 1991; Concannon et al., 1977; Wildt, Panko, Chakraborty, \& Seager, 1979).

The manufacturers of the CLIA prescribe the use of serum. Kutzler et al. (2003) and Volkmann (2006) and Chapwanya et al. (2008) used serum to compare the values of progesterone measured with RIA to those measured with the CLIA. The studies cited earlier in this Introduction show that nine studies report on the use of RIA to measure the values of progesterone in plasma and only two on the values in serum. In the assay brochure, the suppliers of the RIA state that, in humans, the mean value of progesterone in heparinized plasma was almost identical to the mean in serum. The progesterone values measured with RIA in serum of bitches or other species of animals have not been compared to those in plasma.

The values of progesterone in serum that were measured with RIA were compared to those measured in serum with the two-cycle CLIA (Kutzler et al., 2003; Volkmann, 2006). Given that the one-cycle CLIA yields different progesterone values than the two-cycle CLIA (Schmicke et al., 2016) and the substantial amount of published research based on the use of RIA (references cited earlier in this Introduction), there is a need to compare the 
values of progesterone measured with the RIA and the one-cycle CLIA. As the use of RIA to measure the value of progesterone in plasma has been reported more frequently than the use on serum, there is a need to compare the values of progesterone measured in the serum of bitches with the one-cycle CLIA (henceforth simply "CLIA") to those measured in their plasma with RIA.

The aim of this study was to compare the values of progesterone in the plasma of dogs as determined with the RIA and the CLIA for values of progesterone at or below those typically associated with ovulation.

\section{Materials and methods}

The project was approved by the Animal Ethics Committee of the Faculty of Veterinary Science of the University of Pretoria (Projects V071-13, v071-14).

\subsection{Collection of specimens and determination of the concentration of progesterone}

All bitches were hospitalised for routine oestrus observation and artificial insemination. During hospitalization all experimental animals were housed and fed commercial dry pellets twice daily and had access to water ad-lib. All the bitches were taken out twice daily for walks.

Vaginal cytology (Concannon \& Digregorio, 1986) and vaginal speculum examination (Lindsay \& Concannon, 1986) were used to identify pro-oestrus and oestrus in each of 62 bitches (26 English bulldog and 36 Boerboel).

Two vials of blood (BD Vacutainer® Clot activator tube and BD Vacutainer® 170 IU lithium heparin tube, BD Plymouth, UK) were collected at the same time, once every 24 or $48 \mathrm{~h}$ during pro-oestrus and early oestrus from each bitch. Serum and plasma were 
harvested from the respective tubes and stored in cryotubes labelled with unique 4-digit random numbers only and frozen at $-18{ }^{\circ} \mathrm{C}$ until evaluation.

Daily or on alternate days during pro-oestrus and early oestrus, two vials of blood (BD VAC PLAIN glass tube and BD Vacutainer ${ }^{\circledR} 170$ IU lithium heparin glass tube, BD Plymouth, UK) were collected at the same time from each bitch. Serum and plasma were harvested from the respective tubes and stored in cryotubes and frozen at $-18^{\circ} \mathrm{C}$ until evaluation.

The value of progesterone in plasma was determined with RIA (Coat-A-Count ${ }^{\circledR}$ radioimmunoassay; Siemens Health Care Diagnostics Inc. Los Angeles, CA USA) and in serum with CLIA (Immulite ${ }^{\circledR} 1000$ Catalogue number LKPW1); Siemens Medical Solutions Diagnostics, Los Angeles, CA, USA). All determinations were done in duplicate ( 2 replicates simultaneously in the same assay). The mean of the two replicate values determined with RIA and CLIA are referred to as progRIA or progCLIA.

Having used a 100-well RIA kit, progRIA was determined in an initial selection of 41 plasma samples. The seven standards in the kit $(0-127.2 \mathrm{nmol} / \mathrm{L})$ were included in the assay. This process was repeated until 285 progRIAs were determined from which 110 could be selected that were as near as possible to uniformly distributed between 0.5 and $25 \mathrm{nmol} / \mathrm{L}$, which is the range of interest. Using three 100-tube CLIA assays, progCLIA was also determined in each of the 110 serum samples that had been collected at the same time as these 110 selected plasma samples.

Six of the 110 plasma samples - two with progRIAs between 2 and 4 nmol/L, two between 13 and $14 \mathrm{nmol} / \mathrm{L}$ and two between 22 and $23 \mathrm{nmol} / \mathrm{L}$ - were each analysed with RIA on six or seven occasions, using a different assay on each occasion. The serum duplicates of 
these six plasma samples were each analysed on three occasions, using three different CLIA assays.

These 110 progRIAs and progCLIAs, from 18 English bulldogs and 22 Boerboels, were used to compare the agreement between the RIA and CLIA assays.

\subsection{Data analysis}

\subsubsection{Comparing the precision of RIA and Immulite}

The values of progesterone of the two replicates of each of the 110 plasma- or serum samples were used to, respectively, determine the intra assay CV for RIA and CLIA.

The interassay CVs for RIA and CLIA were determined using the progRIAs and progCLIAs of the six plasma- and serum samples each analysed in six or seven and three assays.

The percentage difference between the maximum and minimum progesterone value of two replicates done on the same plasma- (RIA) or serum sample (CLIA) in the same assay were compared using Wilcoxon's rank sum test.

\subsubsection{Assessing the agreement between RIA and Immulite}

The square root of progCLIA was regressed on the square root of progRIA. Squaring both sides of the derived regression equation provided a formula for predicting progCLIA from progRIA.

ProgCLIA, expressed as a percentage of progRIA was regressed on progRIA.

Data analysis was done using Stata 14 (Stata Corp, College Station, TX, USA) and Excel. 


\section{Results}

\subsection{The precision of RIA and CLIA}

The intra- and interassay coefficients of variation were $5.85 \%$ and $8.45 \%$ for RIA and $6.70 \%$ and $9.16 \%$ for CLIA.

The percentage difference in progesterone value between replicates were similar $(p=0.64)$ for RIA and CLIA and fell between $11 \%$ and $31 \%$ with RIA and $13 \%$ and $31 \%$ with CLIA in $25 \%$ of samples (Table 1 ).

Table 1. The percentage difference between the maximum and minimum concentrations of progesterone in 2 replicates simultaneously done on the same plasma or serum sample in the same Coat-A-Count ${ }^{\circledR}$ RIA or Immulite ${ }^{\circledR} 1000$ LKPW1 assay were similar $(\mathrm{P}=0.64)$

\begin{tabular}{lll}
\hline & RIA & Immulite \\
\hline Minimum & 0 & 0 \\
25th Percentile & 3.37 & 2.67 \\
Median & 6.54 & 7.79 \\
75th Percentile & 11.41 & 12.54 \\
Maximum & 31.05 & 30.95 \\
Number of pairs of replicates & 110 & 110 \\
& & \\
\hline
\end{tabular}

\subsection{The agreement between CLIA and RIA}

For 97 of 110 blood samples progImm was lower than progRIA (Figure 1). 


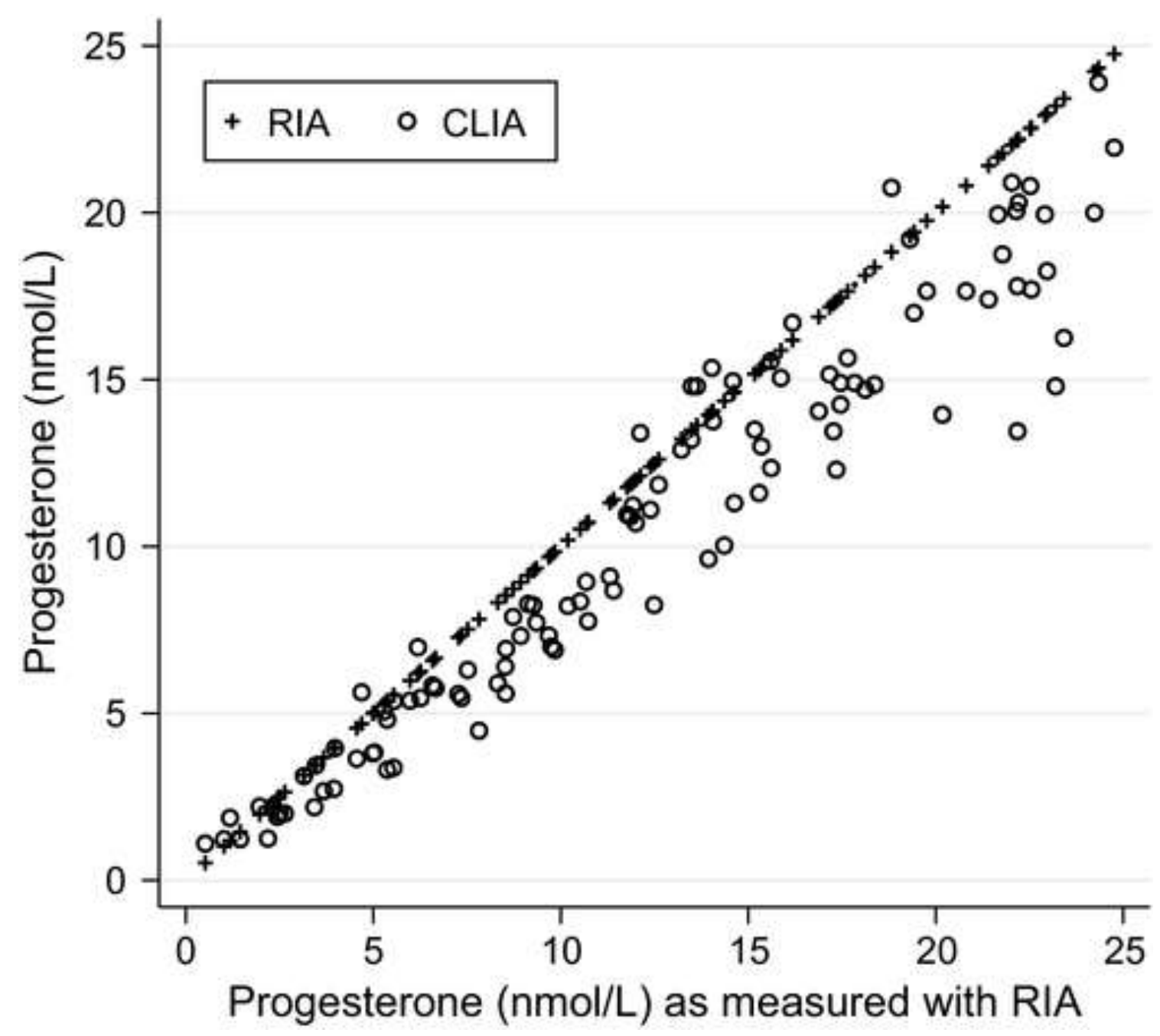

Figure 1. The values of progesterone measured with a CLIA in 110 serum samples and with a RIA in 110 plasma samples — with each pair of serum and plasma samples drawn from the same bitch at the same timeeach plotted against the values measured with the RIA

Figure 2 shows a strong linear dependence of the square root of progImm on that of progRIA ( $\mathrm{R}^{2}$ 0.945, regression coefficient $0.912(95 \%$ CI $0.870-0.953, \mathrm{P}<0.001)$. 


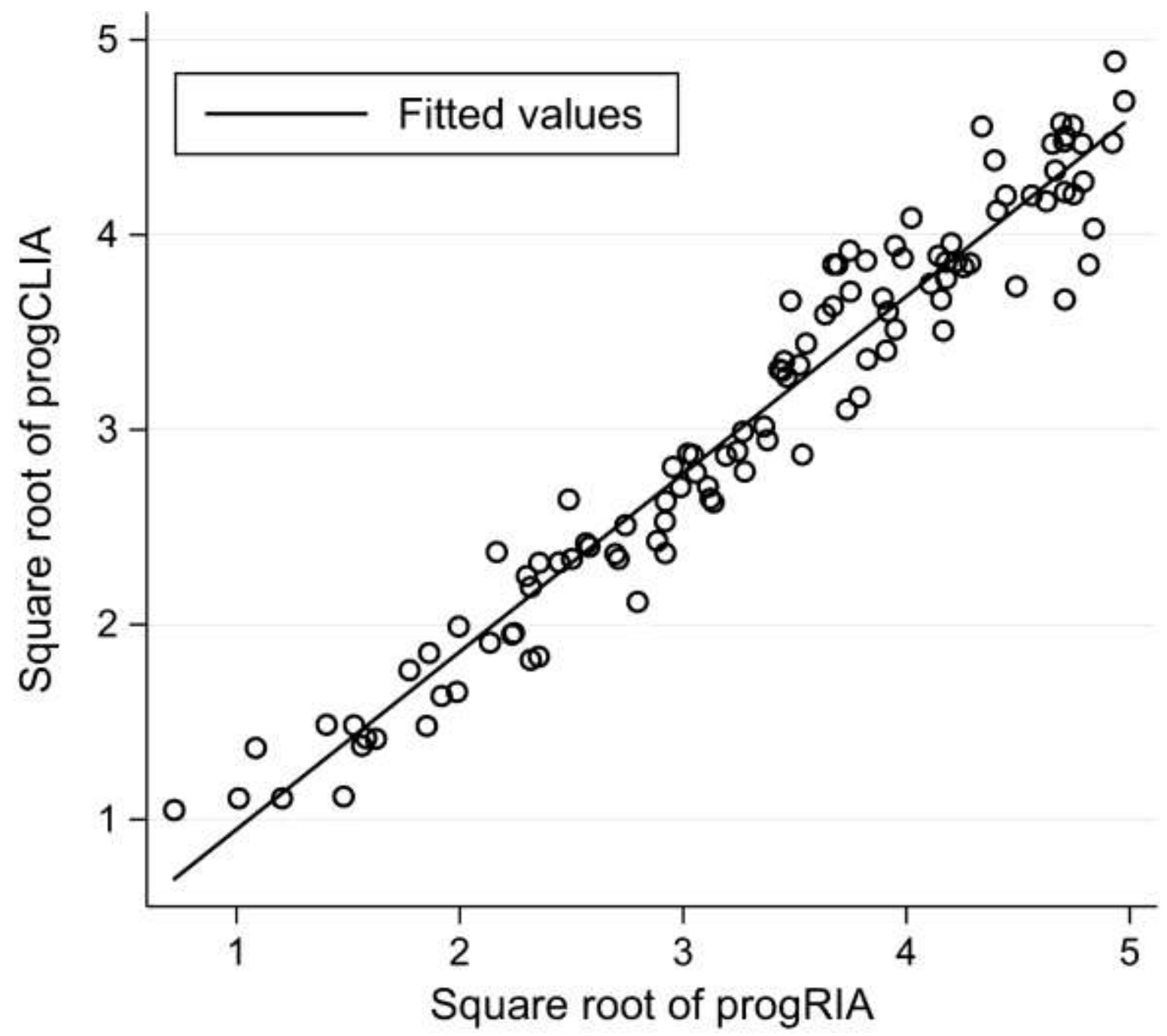

Figure 2. Square roots of the values of progesterone in serum measured with a CLIA (progCLIA) against the square roots of the values of progesterone measured in plasma with a RIA (progRIA), and the regression line $(n=110$ pairs $)$

The model pertaining to the regression was:

progImm $\operatorname{Im}^{0.5}=0.03785+0.91162 \times \operatorname{prog} R I A^{0.5}+$ error

where error has the expected value of zero. Squaring yielded the following equation by which progImm may be estimated from progRIA:

ProgImm $=0.001433+0.06901 \times$ progRIA ${ }^{0.5}+0.831051 \times$ progRIA

This equation yielded estimated values for progImm from $87 \%$ to $90 \%$ of progRIA for progRIAs $\leq 3 \mathrm{nmol} / \mathrm{L}$ and $85 \%$ to $86 \%$ for higher progRIAs. 
ProgImm as a percentage of progRIA was independent of progRIA $\left(\mathrm{R}^{2} 0.00\right.$, slope 0.01 ( $95 \%$ CI -0.38 to 0.40$), P=0.96$ ), with the y-intercept narrowly spread around the mean of $85.2 \%$ (95\% CI 79.71-90.63, $\mathrm{P}<0.001)$. Across the range of progRIA, progImm is on average equal to $85 \%$ of progRIA, but with a large scatter around this mean (95\% CI 58\% to $112 \%$ ) as shown in Figure 3. This mean is similar to the percentages estimated with the regression equation derived from the regression of the square root of progImm on progRIA.

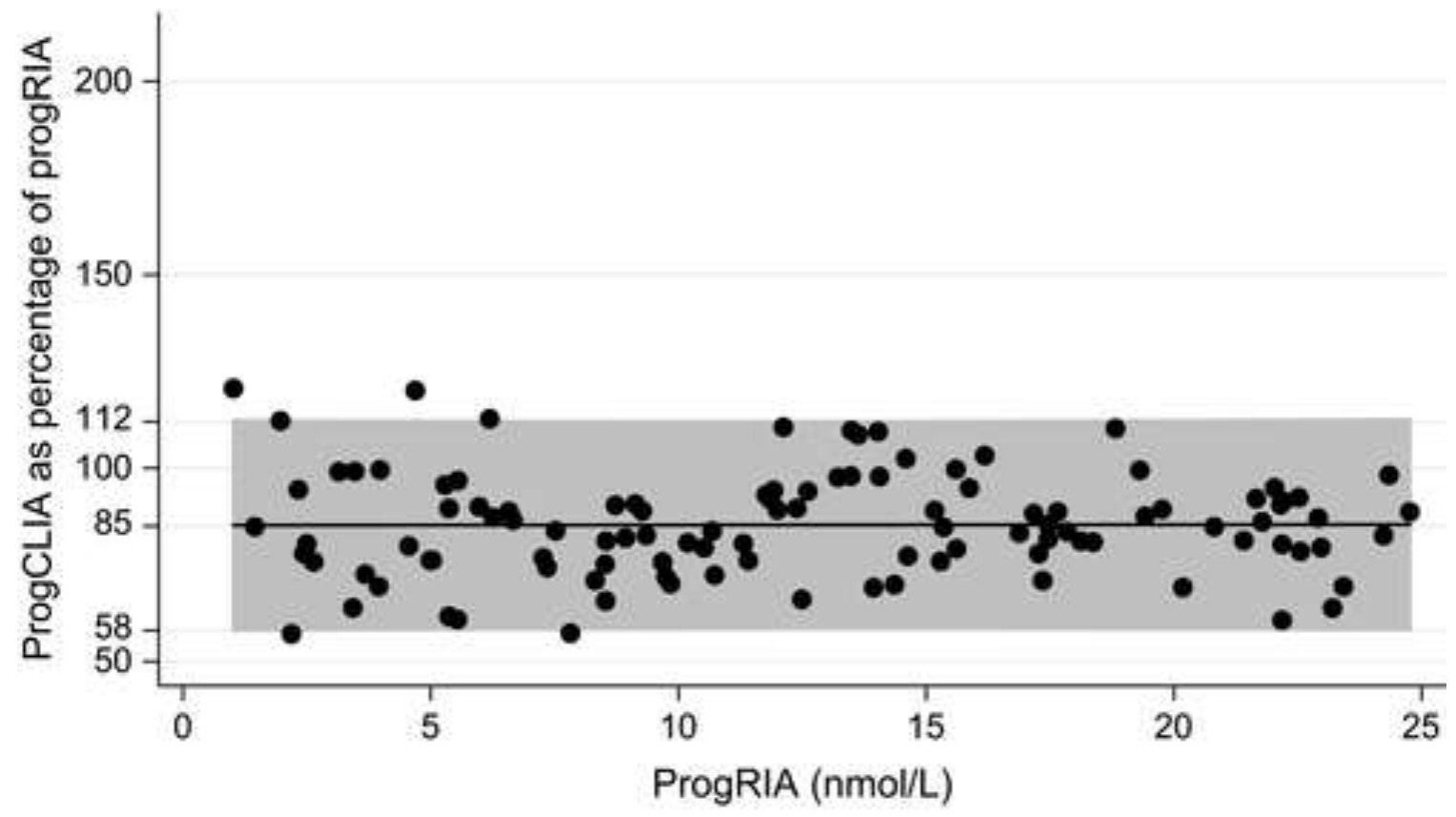

$95 \% \mathrm{Cl}$ for an individual forecast

Fitted values

Two outliers $(210.87 \%$ by $0.52 \mathrm{nmol} / \mathrm{L}$ and $158.5 \%$ by $1.18 \mathrm{nmol} / \mathrm{L}$ are excluded from the regression and $95 \% \mathrm{Cl}$ and not shown

Figure 3. Scatterplot of the values of progesterone measured with a CLIA (ProgCLIA) in serum of bitches, expressed as a percentage of the value measured with RIA in plasma (progRIA), against progRIA $(n=110$ pairs). The linear regression line with the $95 \% \mathrm{CI}($ mean $\pm 1.96 \mathrm{SD})$ for an individual forecast are also shown

\section{Discussion}

\subsection{The precision of the assays}

The intra-assay coefficients of variation found in the current study are similar to the $6 \%$

Okkens et al. (2001) found for the same RIA and the 11\% that Kutzler et al. (2003) 
reported for the two-cycle CLIA and the $7.1 \%$ that Schmicke et al. (2016) reported for the same one-cycle CLIA that was used in the current study. The interassay coefficients of variation found in the current study are similar to the 10.8\% Okkens et al. (2001) found for the same RIA and the $6.7 \%$ and $11.9 \%$ that Kutzler et al. (2003) found with the two-cycle CLIA and the $8.9 \%$ that Schmicke et al. (2016) found with the one-cycle CLIA.

Clinically important decisions in the bitch depend on precise measurements of the value of progesterone in plasma or serum. With the RIA and CLIA, differences as large as $11 \%$ $31 \%$ occur in $25 \%$ of replicates in the same assay. This suggests that samples should be analysed in duplicate to identify large differences between replicate measurements and the assay repeated if a more precise assessment is required.

\subsection{The agreement between progCLIA and progRIA}

On average, progCLIA was $15 \%$ lower than progRIA throughout the range of $0.5-$ $25 \mathrm{nmol} / \mathrm{L}$ in progRIA, with $88 \%$ of progCLIAs being lower than the progRIAs. As with the one-cycle CLIA in the current study, the two-cycle CLIA also yielded lower values of progesterone than the RIA (Kutzler et al., 2003; Volkmann, 2006). Volkmann (2006) found that the average value of progesterone found in serum with the two-cycle CLIA was about one-third lower than that found with the RIA, which seems a larger difference than that found in the current study.

Although the current study shows that progCLIA is on average $85 \%$ of progRIA, there is a wide scatter around this mean, with the $95 \% \mathrm{CI}$ as wide as $28 \%$ percentage points above and $28 \%$ percentage points below the mean. This variability suggests that a clinician should not see a progesterone value obtained in isolation but in the light of other historic, clinical and laboratory findings pertaining to the bitch. 
The current study shows that large differences in progesterone value sometimes occur with replicate analyses in the same assay, suggesting that duplicate analyses are required, especially when high precision is required.

This study used a late opportunity to study and report on the agreement between the longused RIA to the new one-cycle CLIA before the RIA was discontinued in 2014.

\section{Acknowledgements}

The National Research Foundation funded the Coat-A-Count radioimmunoassay kits and the laboratory fees for the determination of values of progesterone. Siemens donated the Immulite kits.

\section{Author contributions}

$\mathrm{J}$ O Nöthling was the author of the scientific protocol and manuscript and performed the statistics. K.G.M. De Cramer was the main person involved in the experimental work and data collection.

\section{Conflicts of interest}

The authors have declared no conflicts of interest.

\section{References}

Baan, M., Taverne, M. A., Kooistra, H. S., de Gier J., Dieleman, S. J., \& Okkens, A. C. (2005). Induction of parturition in the bitch with the progesterone-receptor blocker aglepristone. Theriogenology, 63, 1958-1972.

Badinand, F., Fontbonne, A., Maurel, M. C., \& Siliart, B. (1993). Fertilization time in the bitch in relation to plasma concentration of oestradiol, progesterone and luteinizing hormone and vaginal smears. Journal of reproduction and fertility.Supplement, 47, 63-67. 
Bergeron, L. H., Nykamp, S. G., Brisson, B. A., Madan, P., \& Gartley, C. J. (2013). An evaluation of B-mode and color Doppler ultrasonography for detecting periovulatory events in the bitch. Theriogenology, 79, 274-283.

Chapwanya, A., Clegg, T., Stanley, P., \& Vaughan, L. (2008). Comparison of the Immulite and RIA assay methods for measuring peripheral blood progesterone levels in Greyhound bitches. Theriogenology, 70, 795-799.

Concannon P., Hansel W., \& McEntee K. (1977). Changes in LH, progesterone and sexual behaviour associated with preovulatory luteinization in the bitch. Biology of Reproduction, 17, 604-613.

Concannon, P. W., Butler, W. R., Hansel, W., Knight, P. J., \& Hamilton, J. M. (1978). Parturition and lactation in the bitch: serum progesterone, cortisol and prolactin. Biol.Reprod., 19, 1113-1118.

Concannon, P. W., Hansel, W., \& Visek, W. J. (1975). The ovarian cycle of the bitch: plasma estrogen, LH and progesterone. Biology of Reproduction, 13, 112-121.

Concannon, P. W. (2011). Reproductive cycles of the domestic bitch. Animal Reproduction Science, 124, 200-210.

Fisher L.D. \& Van Belle G. (1993). Biostatistics: a methodology for the health sciences. Wiley-Interscience .

Fontbonne, A. (2008). In vivo ovulation, oocyte maturation and fertilisation in the bitch. Ecole AgroParisTech <NNT : 2008AGPT0010>.

Gerstenberg, C. \& Nöthling, J. O. (1995). The effects of metergoline combined with PGF2alpha treatment on luteal function and gestation in pregnant bitches. Theriogenology, 44, 649-659. 
Groppetti, D., Aralla, M., Bronzo, V., Bosi, G., Pecile, A., \& Arrighi, S. (2015). Periovulatory time in the bitch: what's new to know?: Comparison between ovarian histology and clinical features. Animal Reproduction Science, 152, 108-116.

Hoffmann, B., Kyrein, H. J., \& Ender, M. L. (1973). An efficient procedure for the determination of progesterone by radioimmunoassay applied to bovine peripheral plasma. Hormone Research in Paediatrics, 4, 302-310.

Jeffcoate, I. A. (1993). Endocrinology of anoestrous bitches. Journal of reproduction and fertility.Supplement, 47, 69-76.

Kutzler, M. A., Mohammed, H. O., Lamb, S. V., \& Meyers-Wallen, V. N. (2003). Accuracy of canine parturition date prediction from the initial rise in preovulatory progesterone concentration. Theriogenology, 60, 1187-1196.

Ludewig, R., Kiaei, D., Plouffe, B., Thompson, S., Woods, A., Tan, S. et al. (2012). Validation of a New and Improved Progesterone Assay on the IMMULITE Immunoassay System. Available at http://www.cred.ca/Portals/0/Images/CRED/PDF/2012.

Luz, M. R., Bertan, C. M., Binelli, M., \& Lopes, M. D. (2006). Plasma concentrations of 13,14-dihydro-15-keto prostaglandin $\mathrm{F}_{2}$-alpha (PGFM), progesterone and estradiol in pregnant and nonpregnant diestrus cross-bred bitches. Theriogenology, 66, 1436-1441.

Okkens, A. C., Teunissen, J. M., Van Osch, W., Van Den Brom, W. E., Dieleman, S. J., \& Kooistra, H. S. (2001). Influence of litter size and breed on the duration of gestation in dogs. Journal of reproduction and fertility. Supplement, 57, 193-197.

Reimers, T. J., Lamb, S. V., Bartlett, S. A., Matamoros, R. A., Cowan, R. G., \& Engle, J. S. (1991). Effects of hemolysis and storage on quantification of hormones in blood samples from dogs, cattle, and horses. American Journal of Veterinary Research, 52, 1075-1080. 
Rota, A., Charles, C., Starvaggi Cucuzza, A., \& Pregel, P. (2015). Diagnostic Efficacy of a Single Progesterone Determination to Assess FullГÇÉTerm Pregnancy in the Bitch. Reproduction in Domestic Animals, 50, 1028-1031.

Schmicke, M., Urhausen, C., Wolf, K., Schmidt, S., \& Günzel-Apel, A. R. (2016). [Evaluation of the blood progesterone concentration in the bitch measured by chemiluminescence immunoassay at the day of ovulation]. Tierarztliche Praxis.Ausgabe,K.Kleintiere/Heimtiere, 44.

Steckler, D., Nöthling, J. O., \& Harper, C. (2013). Prediction of the optimal time for insemination using frozen-thawed semen in a multi-sire insemination trial in bitches. Animal Reproduction Science, 142, 191-197.

Van Klaveren, N. J., Kooistra, H. S., Dieleman, S. J., Van Lith, H. A., \& Schaefers-Okkens, A. C. (2001). [The optimal mating time in the bitch based on the progesterone concentration in peripheral blood. A comparison of reliability between three ELISA test kits and a 125-iodine radioimmunoassay]. Tijdschrift voor diergeneeskunde, 126, 680-685.

Volkmann, D. H. (2006). The effects of storage time and temperature and anticoagulant on laboratory measurements of canine blood progesterone concentrations. Theriogenology, 66, 1583-1586.

Wildt, D. E., Panko, W. B., Chakraborty, P. K., \& Seager, S. W. J. (1979). Relationship of serum estrone, estradiol- $17 \beta$ and progesterone to $\mathrm{LH}$, sexual behavior and time of ovulation in the bitch. Biology of Reproduction, 20, 648-658. 